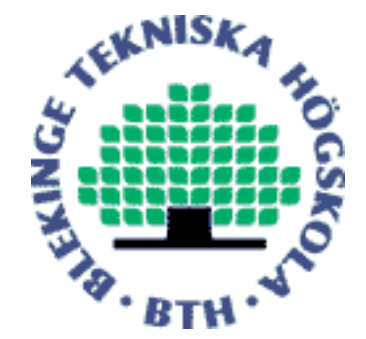

Copyright (C) 2010 IEEE.

Citation for the published paper:

Sub-Frame Crossing for Streaming Video over Wireless Networks

Hussein Muzahim Aziz, Håkan Grahn, Lars Lundberg

7th International Conference on Wireless On-Demand Network Systems and Services

2010 Kranjska Gora

This material is posted here with permission of the IEEE. Such permission of the IEEE does not in any way imply IEEE endorsement of any of BTH's products or services Internal or personal use of this material is permitted. However, permission to reprint/republish this material for advertising or promotional purposes or for creating new collective works for resale or redistribution must be obtained from the IEEE by sending a blank email message to pubs-permissions@iee.org.

By choosing to view this document, you agree to all provisions of the copyright laws protecting it. 


\section{Sub-Frame Crossing for Streaming Video over Wireless Networks}

\author{
Hussein Muzahim Aziz \\ School of Computing \\ Blekinge Institute of Technology \\ Ronneby, Sweden \\ hussein.aziz@bth.se
}

\author{
Håkan Grahn \\ School of Computing \\ Blekinge Institute of Technology \\ Ronneby, Sweden \\ hakan.grahn@bth.se
}

\author{
Lars Lundberg \\ School of Computing \\ Blekinge Institute of Technology \\ Ronneby, Sweden \\ lars.lundberg@bth.se
}

\begin{abstract}
Transmitting a real time video streaming over a wireless network cannot guarantee that all the frames could be received by the mobile devices. The characteristics of a wireless network in terms of the available bandwidth, frame delay, and frame losses cannot be known in advanced. In this work, we propose a new mechanism for streaming video over a wireless channel. The proposed mechanism prevents freezing frames in the mobile devices. This is done by splitting the video frame in two sub-frames and combines them with another sub-frame from different sequence position in the streaming video. In case of lost or dropped frame, there is still a possibility that another half (sub-frame) will be received by the mobile device. The receiving sub-frames will be reconstructed to its original shape. A rate adaptation mechanism will be also highlight in this work. We show that sever can skip up to $50 \%$ of the sub-frames and we can still be able to reconstruct the receiving sub-frame and eliminate the freezing picture in the mobile device.
\end{abstract}

Keywords-streaming video; wireless network; frame splitting; sub-frame crossing; sub-frame merging; rate adaptation;

\section{INTRODUCTION}

Mobile cellular networks provide freedom to the mobile users for calling and receiving calls anywhere and at any time while the mobile users are on the move. Streaming technology delivers video frame over a network from the server to the client in real time. Streaming video is the classical technique for achieving smooth playback of video directly over the network without downloading the entire file before playing the video [1]-[3]. Streaming video requires high reliability with a low bounded jitter (i.e. variation of delays) and reasonably high transmission rate [4].

Real-time video streaming requires a steady flow of information and delivery of frame packets according to their deadline; wireless radio networks have difficulties to provide such reliable service [5]. However, the transmission errors in forms of packet delay, jitter (packet inter-arrival variations), and packet loss can have major impact on the end user experience.

This is particularly true in a cellular network environment where the channel condition can vary dramatically due to fading and other network effects [6].

\footnotetext{
This work was supported by the Swedish Knowledge Foundation.
}

Bandwidth is one of the most critical resources in wireless networks, and thus, the available bandwidth of wireless networks should be managed in an efficient manner [7]. Therefore the transmission rate of streaming video should be reduced according to the bandwidth of the communication networks [8][9]. Network adaptation refers to how many network resources (e.g., bandwidth) a video stream should utilize for video content, resulting in designing an adaptive streaming mechanism for video transmission [10]. To stream video, it is desirable to adjust the transmission rate according to the perceived congestion level in the wireless network to maintain a suitable loss level and fairly shared bandwidth with other connections. Furthermore, it's favourable for the streaming video to be aware of the transmission level in the wireless channel in order to obtain good streaming quality by appropriate error protection.

Aziz et al. [11], present a mechanism to split the video frame into two sub-frames and transmit it over two wireless channels in a cellular network. After the two video streams have been received by the mobile device, a checking procedure will used to identify the missing sub-frames. The checking procedure is needed to identify the missing subframe and to reconstruct the available sub-frame. The reconstruct mechanism is based on taking the average of the neighbouring pixels to replace the missing sub-frame to get fully frame shape, but the main limitation for their work is transmitting each video frame over two wireless channels.

To overcome the transmission of each frame over two wireless channels, a sub-frame crossing mechanism is proposed based on frame splitting mechanism in [11]. It will split the frame in two sub-frames, and combine the sub-frame with another sub-frame from a different sequence position and transmitted as a single frame over a single wireless channel. In case of frame losses or frames corruption from the streaming video, there is still a possibility that one of the sub-frame will be received by the mobile device. The receiving sub-frame will be reconstructed to its full frame shape.

The remainder of this paper is organized as follows. Section II explains the proposed mechanism for real time video streaming by using sub-frame crossing mechanism and transmitted over single channel, while the rate adaption are presented in Section III. The discussion and the conclusion of the proposed mechanism will be presented in Section IV. 


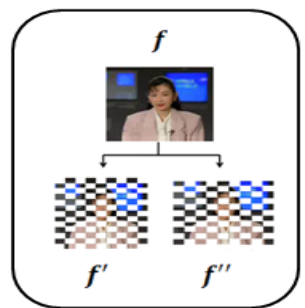

Figure1. Frame split.

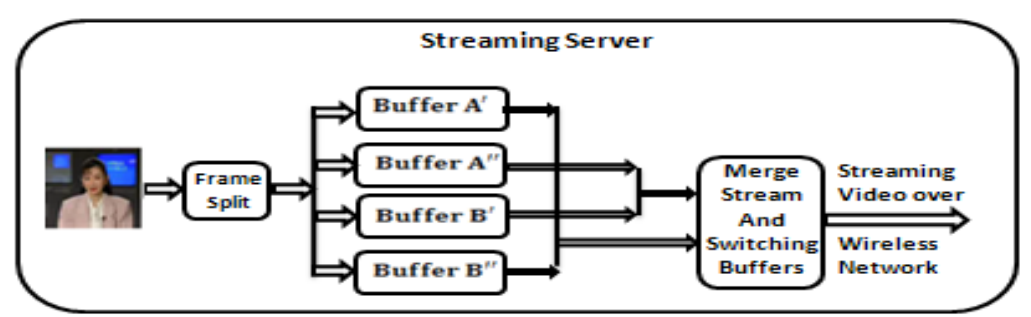

Figure 2. Streaming crossing sub-frames over wireless channels.

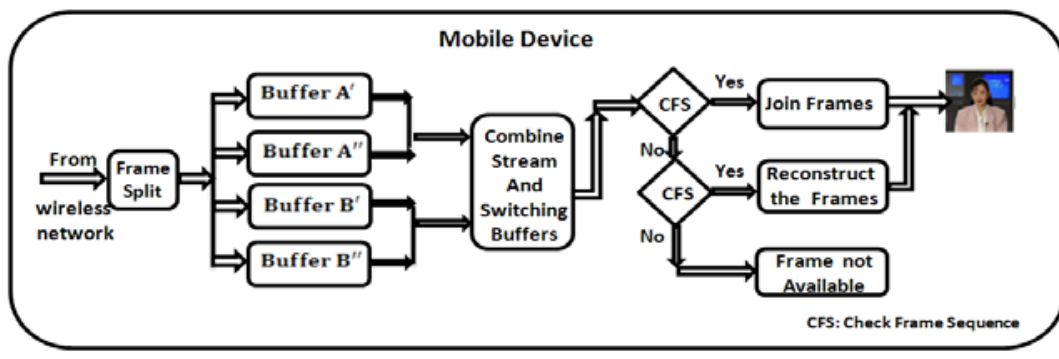

Figure 3. Receiving the streaming sub-frames crossing in the mobile device.

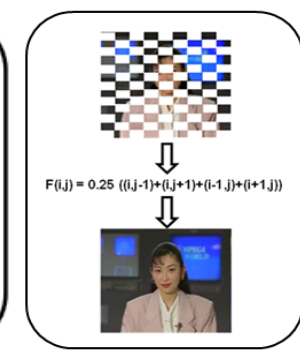

Figure 4. Reconstruct frame.

\section{THE PROPOSED TECHNIQUE}

Mobile video streaming is characterized by low resolutions and low bit rates. The bit rates are limited by the capacity of UMTS radio bearer and the restricted processing power of mobile terminals. The commonly used resolutions are Quarter Common Intermediate Format (QCIF, 176x144 pixels) for cell phones [12]. Mobile real time application like video streaming suffers from high loss rates over the wireless network [13] and the effect of that the mobile users may notice some sudden stop during the playing video, the picture is momentarily frozen. Frozen pictures could occur if the video frames have been delayed or been dropped.

The proposed mechanism is based on the way of packetizing the video frame and transmitted over a single wireless channel and this can be done by splitting the video frame into two sub-frames based on the pixel splitting technique [11] to create two sub-frames out of each frame, where one sub-frame contains the even pixels and another contains the odd pixels as shown in Fig. 1[11].

The mobile client's request the connection to the server, the server will start streaming by splitting each frame into two sub-frames which will be queued in different buffers according to the frames sequence number and to its contains.

In this study, we will assume that the transmission rate is 30 frames/second. The first 15 frames $\left(\mathrm{f}_{1}, \mathrm{f}_{2}, \mathrm{f}_{3, \ldots}, \mathrm{f}_{15}\right)$ will be queued in two buffers and each buffer will hold 15 sub-frames as an example Buffer $\mathrm{A}^{\prime}=\left(\mathrm{f}^{\prime}{ }_{1}, \mathrm{f}_{2,}^{\prime} \mathrm{f}_{3, \ldots}^{\prime}, \mathrm{f}_{15}^{\prime}\right), \mathrm{A}^{\prime \prime}=\left(\mathrm{f}^{\prime \prime}{ }_{1,} \mathrm{f}^{\prime \prime}{ }_{2}\right.$, $\left.\mathrm{f}^{\prime \prime}{ }_{3, \ldots} \mathrm{f}^{\prime \prime}{ }_{15}\right)$, while the next 15 frames $\left(\mathrm{f}_{16}, \mathrm{f}_{17}, \mathrm{f}_{18, \ldots}, \mathrm{f}_{3,0}\right)$ will also be queued in two buffers as $\mathrm{B}^{\prime}=\left(\mathrm{f}_{16}, \mathrm{f}_{17,}^{\prime} \mathrm{f}_{18, \ldots, \mathrm{f}_{30}}\right), \mathrm{B}$ $=\left(\mathrm{f}^{\prime \prime}{ }_{16}, \mathrm{f}^{\prime \prime}{ }_{17}, \mathrm{f}^{\prime \prime}{ }_{18}, \ldots, \mathrm{f}^{\prime \prime}{ }_{30}\right)$, as shown in Fig. 2.
The crossing mechanism between two sub-frames from different buffers and from different sequence position will be combined with each other and will create as a normal frame and as an example $\left\{\left(\mathrm{f}_{1,}^{\prime} \mathrm{f}_{16}^{\prime \prime}\right),\left(\mathrm{f}_{2}^{\prime}, \mathrm{f}^{\prime \prime}{ }_{17}\right), \ldots, \ldots \ldots,\left(\mathrm{f}_{14}^{\prime}, \mathrm{f}^{\prime \prime}{ }_{29}\right)\right.$, $\left.\left(\mathrm{f}_{15}^{\prime}, \mathrm{f}_{30}^{\prime \prime}\right),\left(\mathrm{f}_{16}^{\prime}, \mathrm{f}_{1}{ }_{1}\right), \ldots,\left(\mathrm{f}_{29}^{\prime}, \mathrm{f}_{14}^{\prime \prime}\right),\left(\mathrm{f}_{30,} \mathrm{f}_{15}\right)\right\}$.

This will be applied to the entire video frame and will be transmitted over a single channel. The reason behind that, if there is a lost or dropped frame from the streaming video, the effect will be on two sub-frames from two different frames that are in different positions from the streaming sequence.

After each frame has been received by the mobile device a splitting frame technique will applied. The sub-frames will be hold in different buffers and according to the way they have been split at the server side as shown in Figure 3. The subframe will distributed to the relevant buffers and a combination of the sub-frames according to their sequence will be considered with buffers switching to create frame sequences of the stream.

A frame checking sequence will take place after the combination between the sub-frames that are related to the original frame. A checking procedure will used to check the availability of the sub-frames, as an example, the first check frame sequence (CFS), will check whether the both subframes that are related to the same frame are available or not. If both of them are available then a join mechanism will be applied to return the frame to its original. In the case when there is a network interruption, e.g., if the sub-frames are corrupted and will be unreadable by the decoder or the subframes are dropped, the second CFS will check if there is at least one sub-frame available or not. 
If it's not available then we considered that the frame is dropped from the frame sequence. If there is at least on subframe are available, the reconstruction of the sub-frame will take place in the mobile device by taken the average of the neighboring pixel to replace the missing pixel and to get the normal frame shape and play it on the mobile screen with acceptable quality level, as shown in Figure 4 [11]. While the experimental study for the sub-frame crossing mechanism has been tested according to International Telecommunication Union (ITU-R) [14] and under different network condition and for different motion video and we get similar results as in [11].

\section{RATE ADAPTION}

Rate adaptation for streaming video is regarded as a necessary mechanism in order to handle the network conditions, and fluctuations of available network bandwidth. During the interactive mode, the server rate is adapted to the requirement of the available bandwidth in the wireless network.

The adaption rate for the sub-frame crossing mechanism should be considered carefully to avoid skipping the subframes that belonging to the same video frame and with the consideration of the network bandwidth and network interruption to the streaming video. This can be done by not considering the combination of the two sub-frame and transmitting only one half of the frame (any sub-frame) to the mobile device and according to the following percentages:

- $10 \%$ adjustment: we will skip every $5^{\text {th }}$ sub-frames from buffer $A^{\prime}$ and $B^{\prime}$.

- $\quad 25 \%$ adjustment: we will skip every even sub-frames from buffer $A^{\prime}$ and $B^{\prime}$.

- $50 \%$ adjustment: we will skip all the sub-frames from buffer $A^{\prime}$ and $B^{\prime}$.

The rate adaptation mechanism is needed to adjust the transmission rate based on the congestion level. The server will adjust the transmission rate by skipping the sub-frames that are not related to each other and the skipping rate limits shouldn't cross $50 \%$ from streaming video to avoid discarding the sub-frames that are related to the same video frame.

\section{DISCUSSION AND CONCLUSIONS}

The high error rate and network congestion raises a number of challenging issues for streaming video over unreliable wireless network. Such issues include techniques to ensure a continuous display at the mobile device in spite of errors in the received video. For wireless networks in particular, the available channel bandwidth varies with time that can potentially result in lost frames or frames arriving much later than its deadline, resulting in garbled frame.

Sub-frame crossing mechanism is proposed to distribute the sub-frames in different positions in the streaming sequence by combine it with another sub-frames. The idea behind that is to avoid losing the complete frame and allow at least half of the frame (sub-frame) to be received by the mobile device.

The proposed mechanism is based on the limitation on [11], where the analysis comparison between the two scenarios is presented in Table 1.
The proposed mechanism is based on packetizing the video frames and transmitted over a single channel. The extra implement delay time is needed for the sub-frame to merge with another sub-frame from different position in the server with the amount of time is needed in the mobile device to combine the sub-frame to the original frame shape is 1 second. While the discard frames rates should be limited to $50 \%$ from the amount of transmission rate been streamed from the server.

Table I: IMPLEMENTATION COMPARISON

\begin{tabular}{|c|c|c|}
\cline { 2 - 3 } \multicolumn{1}{c|}{} & Proposed & In [11] \\
\hline No. of Channel(s) & 1 & 2 \\
\hline Overhead & Normal & Double \\
\hline $\begin{array}{c}\text { Extra Delay Time } \\
\text { Implementation }\end{array}$ & 1 Sec. & 2 Sec. \\
\hline
\end{tabular}

Streaming the sub-frame over tow channels scenario [11] will increase the overhead to double; while the implementation delay time been applied is 2 seconds. Implementing the delay time is to avoid the transmission of the same sub-frame at the same time under the same network condition and this will minimize the effects on the same frame. While the combination between sub-frames will take place after the mobile devices start receiving the same amount of sub-frames from both channels. While the main strength is that if the amount of skipped frames are high it's still there is a possibilities that we could reconstruct the missing sub-frame.

Our proposed mechanism appears to provides a promising direction for eliminating the freezing picture in the mobile screen, that been caused by the missing frames from the streaming sequence. Adjusting the amount of frames to be transmitted according to the changes in the bandwidth is highly needed to reduce the amount of frames that needs to be send to the mobile device under congested network. However, the quality of the receiving video is degraded.

\section{REFERENCES}

[1] B. Guangwei, and W. Carey," The effects of mobility on wireless media streaming performance," In Proceedings of Wireless Networks and Emerging Technologies. Banff, AB, Canada, WNET `04, pp: 596-601, July 8-10, 2004.

[2] C. Xiaozhen, B. Guangwei, and W. Carey, "Media streaming performance in a portable wireless classroom network," In Proceedings of IASTED European Workshop on Internet Multimedia Systems and Applications. EuroIMSA '05. Grindelwald, Switzerland, pp: 246-252, Feb. 21-23, 2005.

[3] H. Zhu, I. H. Wang, and B. Chen, "Bandwidth scalable source-channel coding for streaming video over wireless access networks," In Proceedings of Wireless Networking Symposium. WNCG' 03. Austin, Texas, Oct. 26-28, 2003.

[4] C. Y. Hsu, A. Ortega, and M. Khansari, "Rate control for robust video transmission over burst-error wireless channels," IEEE Journal on Selected Areas in Communications. vol. 17, no. 5, pp: 756 - 773, 1999.

[5] X. Zhu, and B. Girod, "Video streaming over wireless networks," In Proceedings of European Signal Processing Conference. EUSIPCO 07. Poznan, Poland, pp: 1462-1466, Sept. 3-7, 2007.

[6] R. Weber, M. Guerra, S. Sawhney, L. Golovanvsky, M. Kang, “ Measurement and analysis of video streaming performance in live 
UMTS networks," In Proceedings of WPMC'06 Conference, San Diego, CA, USA, pp: 1-5, Sept. 17-20, 2006.

[7] J.-Y. Chang, and H-L Chen," Dynamic-grouping bandwidth reservation scheme for multimedia wireless networks," IEEE Journal on Selected area in Communications, vol. 21, no. 1, pp. 1566 - 1574, 2003.

[8] G-R. Kwon, S-H. Park, J-W. Kim, and, S-J. Ko,” Real-time R-D optimized frame-skipping transcoder for low bit rate video transmission," $6^{\text {th }}$ IEEE International Conference on Computer and Information Technology. Seoul, Korea, Sept. 20-22, 2006.

[9] H. Luo, M-L. Shyu, and S-C. Chen, "An end-to-end video transmission framework with efficient bandwidth utilization," In Proceedings of the IEEE International Conference on Multimedia and Expo. ICME 2004. Taipei, Taiwan, R.O.C., pp. 623-626, June 27-30, 2004.

[10] F. Yang, Q. Zhang, W. Zhu, and Y-Q Zhang, "Bit allocation for scalable video streaming over mobile wireless Internet," Twenty-third Annual Joint Conference of the IEEE Computer and Communications Societies. pp: 2142-2151, March 7-11, 2004.
[11] H. M. Aziz, H. Grahn, and L. Lundberg, "Eliminating the freezing frames for the mobile user over unreliable wireless networks," In the Proceedings of the $6^{\text {th }}$ International Conference on Mobile Technology, Applications and Systems. ACM Mobility Conference, France, Nice, Sept. 2- 4, 2009.

[12] M. Ries, O. Nemethova, and M. Rupp, "Performance evaluation of mobile video quality estimators," In Proceedings of the European Signal Processing Conference, EUSIPCO'07. Poznan, Poland, pp: 159-163, Sept. 3-7, 2007.

[13] T. Nguyen, P. Mehra, and A. Zakhor, "Path diversity and bandwidth allocation for multimedia streaming," In Proceedings of the International Conference on Multimedia and Expo. ICME `03. Baltimore, Maryland, pp: 1-4, 2003.

[14] International Telecommunication Union. Methodology for the subjective assessment of the quality of television pictures. ITU-R, Rec. BT.500-11, 2002. 